\title{
The participation of singlet oxygen in a photocitotoxicity of extract from amazon plant to cancer cells
}

Anna V. Tcibulnikova, Igor A. Degterev, Valery V. Bryukhanov, Matheus Mantuanelli Roberto, F. D. Campos Pereira, et al.

Anna V. Tcibulnikova, Igor A. Degterev, Valery V. Bryukhanov, Matheus Mantuanelli Roberto, F. D. Campos Pereira, M. A. Marin-Morales, Vasily A. Slezhkin, llya G. Samusev, "The participation of singlet oxygen in a photocitotoxicity of extract from amazon plant to cancer cells," Proc. SPIE 10456, Nanophotonics Australasia 2017, 104563E (2 January 2018); doi: $10.1117 / 12.2283317$ 


\title{
The participation of singlet oxygen in a photocitotoxicity of extract from amazon plant to cancer cells
}

\author{
V. Anna Tcibulnikova ${ }^{\mathrm{a} *}$, A. Igor Degterev ${ }^{\mathrm{a}, \mathrm{b}}, \mathrm{V}$. Valery Bryukhanov ${ }^{\mathrm{a}}$, Matheus Mantuanelli Roberto ${ }^{\mathrm{c}, \mathrm{d}}$, \\ F.D. Campos Pereira ${ }^{\mathrm{c}, \mathrm{e}}$, M. A. Marin-Morales ${ }^{\mathrm{c}}$, A. Vasily Slezhkin, ${ }^{\mathrm{a}}$, G. Ilya Samusev ${ }^{\mathrm{a}}$ \\ åmmanuel Kant Baltic Federal University, \\ *Interdisciplinary reference centre: FunMagMa (Project 5 Top100), \\ 14 A.Nevskogo St., Kaliningrad, Russian Federation, 236016; \\ ${ }^{b}$ Universidade Federal do Acre, Biotecnologia e Biodiversidade, Rio Branco/AC, Brazil, 69920-900;

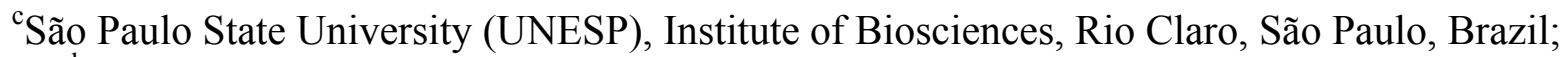 \\ ${ }^{\mathrm{d}}$ Hermínio Ometto University Center (FHO|UNIARARAS), Araras, São Paulo, Brazil; \\ ${ }^{e}$ Paulista University (UNIP), Limeira, São Paulo, Brazil.
}

\begin{abstract}
We have been searching for new photosensitizers (PS) for photodynamic therapy (PDT) of cancer based on extracts from Amazonian plants since 2009. In this paper, we demonstrate that, under certain conditions, the extract from fruits of the Amazonian palm Euterpe oleraceae (popular name Açaí) can serve as a PS for PDT treatment of murine breast cancer cells (4T1 cell line). We have been first to show directly that the photodynamic effect of plant PS is due to singlet oxygen.
\end{abstract}

Keywords: PDT, PS, cancer cells, amazon Palm Tree, plant extract, singlet oxygen, laser, UV-Vis and fluorescent spectra, nanoemulsion, MTT method

\section{INTRODUCTION}

The PDT is a minimally invasive and promising modality to combat cancer. Advantage of this approach is less destructive activity compared to chemotherapy and radiotherapy [1]. In principle, the PDT process is based on the excitation of molecular oxygen into a cancer cell with a red light photon and, thereby, turning it into a reactive product $\left(\mathrm{OH},{ }^{1} \mathrm{O}_{2}\right.$, etc.) initiating oxidative stress and, ultimately, destroying the target cell. However, the small size of both participants in the process makes it extremely unlikely that they will meet.

Despite the significant progresses of drug design, herbal drugs remaining a potential source of pharmacology. The crucial step in the hunting for new drugs is the search for plant extracts that possessing medicinal properties. An Amazon ecosystem contains the great part of the botanical biodiversity of our Planet. However, very few natural pharmaceutics have reached the markets. Many recent papers have documented the phytochemical and pharmacological bases for the use of palms (Arecaceae) in ethnomedicine [2,3]. Early publications were based almost entirely on interviews that solicited local knowledge. We have recently demonstrated that acai oil associated with nanoemulsion is a potential tool for fighting melanoma B16 [4]. Although in the work cited, we assumed that the singlet oxygen is responsible for the photodynamic effect, there was no evidence for this. In that work, we confined ourselves to demonstrating the generation of the ROS in the process of irradiating Asai oil with red light. Therefore, in this paper we compared the PDT effect of the extract from Acai oil with a direct measurement of the singlet oxygen generation in this extract upon irradiation.

\section{MATERIALS AND METHODS}

Oil from Euterpe oleraceb (Açaí) fruit were obtained by mechanical pressing of the seeds of a (popular name açaí).

Subsequent to this, extraction with hot water from the oil was carried out and the resulting extract was dried in a vacuum.

Nanophotonics Australasia 2017, edited by James W. M. Chon, Baohua Jia, Proc. of SPIE Vol. 10456, 104563E - (c) 2018 SPIE · CCC code: 0277-786X/18/\$18 - doi: 10.1117/12.2283317 
Cancer cell culture of metastatic breast cancer (4T1) were maintained in $75 \mathrm{~cm}^{3}$ culture dishes with DMEM medium supplemented with $10 \%$ of fetal bovine serum, $100 \mathrm{U} / \mathrm{mL}$ penicillin, and streptomycin $(100 \mu \mathrm{g} / \mathrm{mL})$. Cells were maintaining at: $80 \%$ humidity, $5 \%$ of $\mathrm{CO}_{2}$ and $370{ }^{\circ} \mathrm{C}$.

The extract photocytotoxicity (the photodynamic effect) to $4 \mathrm{~T} 1$ cells were measured in the dark and under illumination with red light of laser (MMoptics, Sao Carlos, Brazil). Suspensions of 4T1 was irradiated in culture wells of a 96-well plate. For the cell viability evaluation, three energies densities were used. As the culture is well well-rounded, $0.35 \mathrm{~cm}^{2}$, the energies densities after 1,3 , and 9 minutes of laser irradiation, was similar to $6.85 ; 20.57$; and $61.71 \mathrm{~J} / \mathrm{cm}^{2}$ respectively. The red laser light with emission wavelength of $660 \mathrm{~nm}$ was applied for a period of time (30 seconds to 9 minutes) to the experimental groups containing cell culture and extract. At the end of the experiments, a solution of PBS was substituted by cell culture. The MTT method [5,6] served to measure cytotoxicity both in dark and under illumination.

For generation singlet oxygen spectra the $6,6 \mathrm{mg}$ of the dry extracts of Açaí were dissolved in $10 \mathrm{ml}$ water solution. The absorption spectra of extracts solution were recorded on spectrophotometer (Shimadzu, Japan). The fluorescence steadystate and time-resolved measurements were carried out with Fluorolog-3 optical device (Horiba, Japan-France). This device is equipped with two detectors for working in visible and infrared regions. The radiation source of this device is a Xe lamp. The picosecond diodes (NanoLed) wavelength $\lambda=405 \mathrm{~nm}(\tau<200 \mathrm{ps})$ was used to record the extract fluorescence lifetimes in visible region. To record spectrum and the emission lifetime of the singlet oxygen in Acai ethanol solution was used pulsed Xe lamp as a source and a cooled Solid State IR-detector with a PS/TC-1 controller.

\section{RESULTS AND DISCUSSION}

The search for potential PS for PDT among extracts from Amazonian plants begins with the study of their absorption spectra. The studied extract from the fruit of the Amazon palm Euterpe oleracea corresponds to the basic requirement for a candidate for PS for PDT, namely, absorption in a resonance region for molecular oxygen - 600-800 nm (Fig. 1).

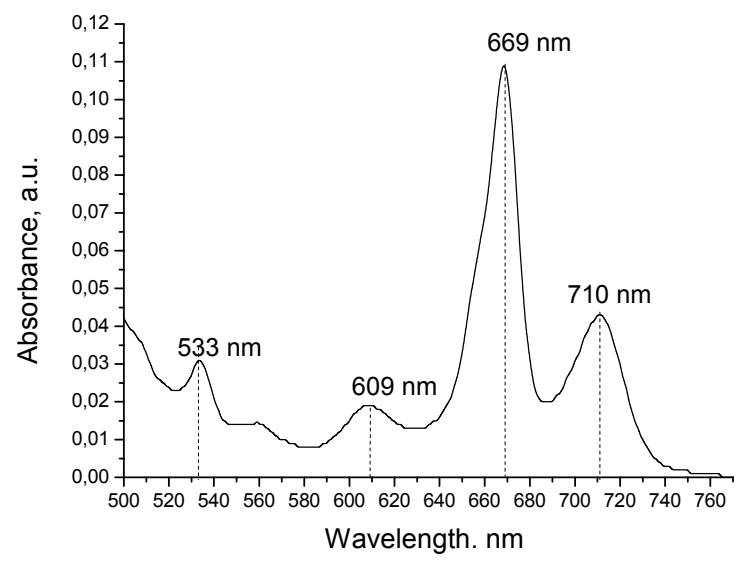

Fig. 1 Absorption Spectrum of a Aqueous Extract from Fruit of Palm Euterpe oleracea in a UV-Vis region.

Are clearly visible peaks in the region from 600 to $800 \mathrm{~nm}$

Thus, according to this parameter, the water extract from the fruit of Açaí meets our hopes as a candidate for PDT and we were tested it for phototoxicity against the cancer cells of the line 4T1. The results of the test are shown in Fig. 2. 


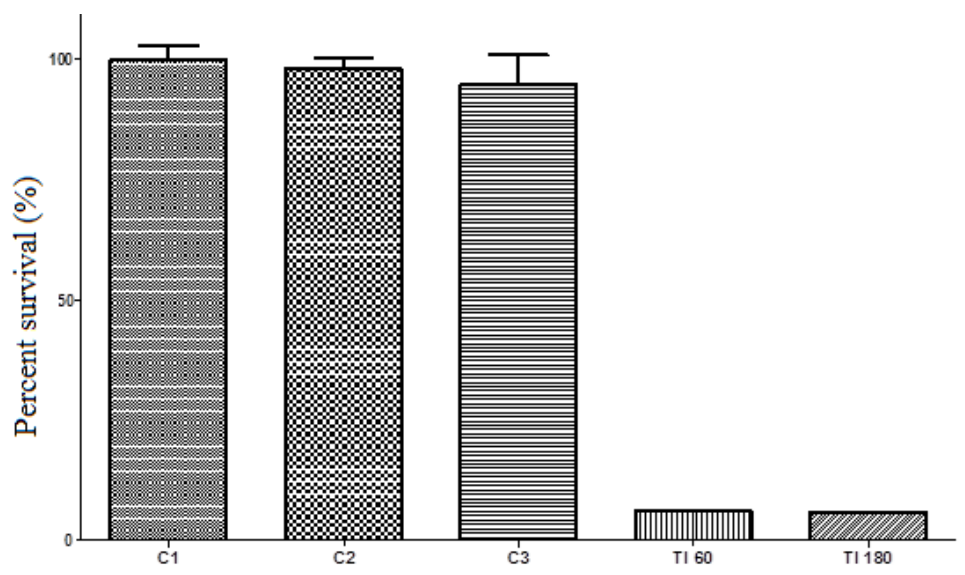

Figure 2. Photodynamic effect of oil extract from Extract from Fruit of Palm Euterpe oleracea against 4T1 cell (mice breast cancer)

Controls: $\mathrm{C} 1$ - the cells were incubated for 2 hours in the dark without the presence of oil (extract); 2 - the cells were incubated for 2 hours in the dark in the presence of respective concentrations of oil (extract); $\mathrm{C} 3$ - the cells were incubated for 2 hours in the dark without the presence of oil (extract) and then illuminated with light of red laser for 4 $\min \left(24 \mathrm{~J} / \mathrm{cm}^{2}\right)$;

$\mathrm{TI}$ - time of illumination (sec) of samples containing both the cells and the extract (oil) with light of red laser, in parentheses the energy of light received by cells during the exhibition. Concentrations: Euterpe oleracea $-1,16 \mathrm{mg} / \mathrm{mL}$, Arrabidea chica $-0,075 \mathrm{mg} / \mathrm{mL}$.

Fluorescence spectra under excitation wavelength $\lambda=530 \mathrm{~nm}$ and $\lambda=420 \mathrm{~nm}$ are shown in Fig. 3

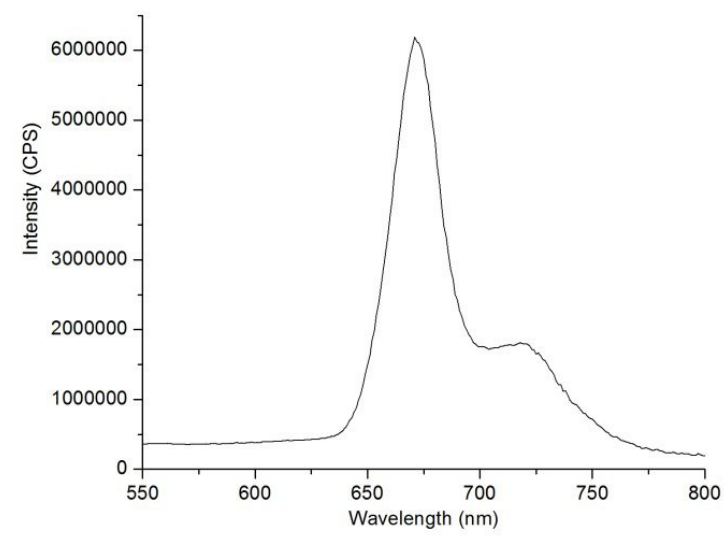

a)

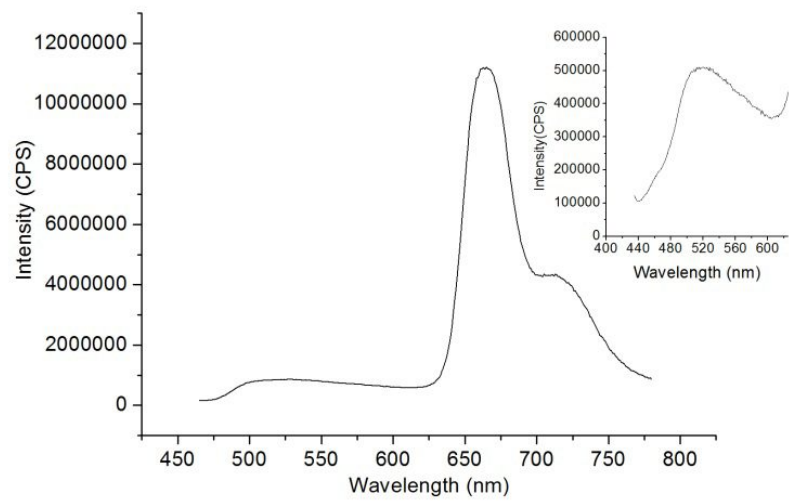

b)

Fig.3 The Açaí fluorescence spectrum when excited at $530 \mathrm{~nm}$ (a) and $420 \mathrm{~nm}$ (b).

According to the data of the absorption analysis, a wide range of wavelengths $(400-540 \mathrm{~nm})$ can excite the fluorescence of molecules of the Açaí extract. As can be seen from Fig. 3b, the intensity of the second maximum (at $\lambda=670 \mathrm{~nm}$ ) upon excitation with a wavelength of $420 \mathrm{~nm}$ is much higher than the emission intensity at $670 \mathrm{~nm}$ with $530 \mathrm{~nm}$ excitation (approximately 2-fold).

At the same time, when $530 \mathrm{~nm}$ was excited, there was no fluorescence at $515 \mathrm{~nm}$. We believe that this increase in the intensity of fluorescence can be due to the transfer of energy between the components of one extract by some mechanism. Further, the fluorescence lifetimes of the molecules of the given extract were measured at the two 
wavelengths

under

study

when

NanoLed-405

$\mathrm{nm}$

was

excited.

The kinetics of attenuation of Açaí luminescence is shown in Fig. 4.

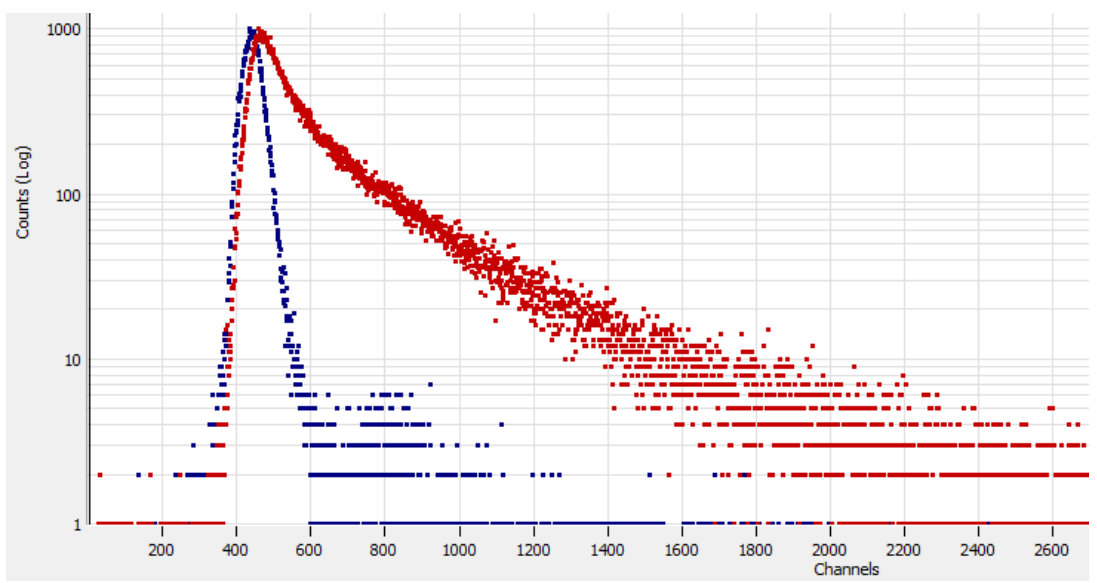

(a)

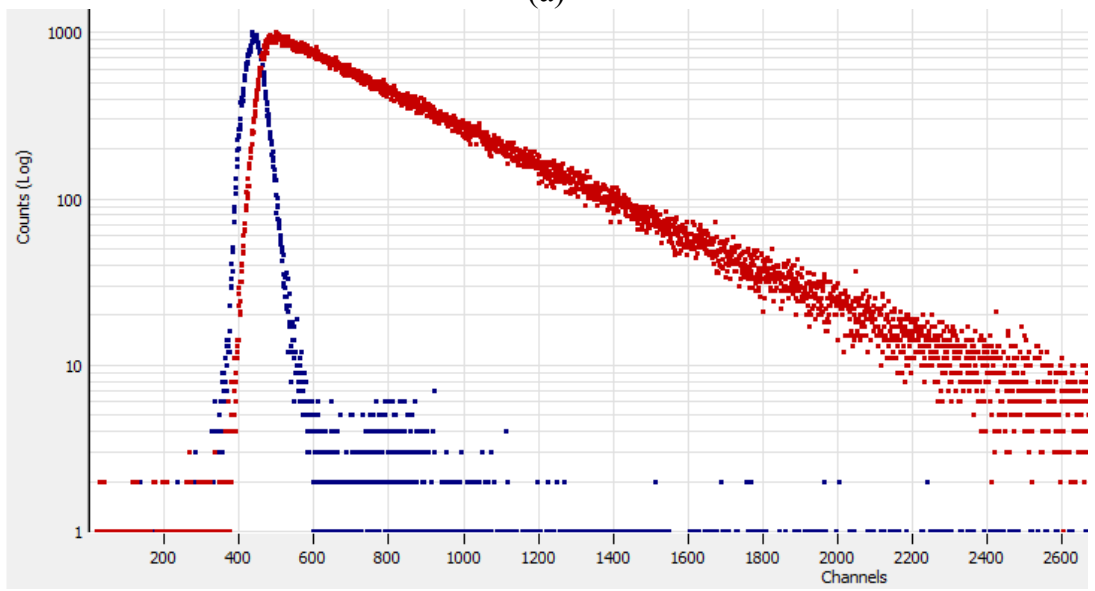

(b)

Fig.4. The kinetics of Açaí fluorescence decay on wavelengths registration $530 \mathrm{~nm}$ (a) and $420 \mathrm{~nm}$ (b).

It should be noted that the duration of the luminescence of the second maximum exceeds the duration of the luminescence of the first maximum, which may be due to energy degradation over the vibrational sublevels of the singlet-excited states.

The biexponential model was used for experimental curves:

$$
I(t)=A+B_{1} \cdot e^{t / T_{1}}+B_{2} \cdot e^{t / T_{2}}
$$

and the average lifetime:

$$
<t>=\frac{B_{1} \cdot T_{1}+B_{2} \cdot T_{2}}{B_{1}+B_{2}} .
$$

where A, B1, B2 - kinetic parameters.

The results of kinetic measurements were presented in Table 1. 
Table 1. Approximation parameters of fluorescence kinetics decay of Açaí as biexponential function and average lifetime of fast fluorescence for two detection wavelengths

\begin{tabular}{|c|c|c|}
\hline $\begin{array}{c}\text { Kinetic } \\
\text { parameters }\end{array}$ & $\boldsymbol{\lambda}_{\mathbf{e m}}=\mathbf{5 0 0} \mathbf{n m}$ & $\boldsymbol{\lambda}_{\mathbf{e m}}=\mathbf{6 7 0} \mathbf{n m}$ \\
\hline $\mathrm{T} 1, \mathrm{~ns}$ & 0.65 & 0.26 \\
\hline $\mathrm{T} 2, \mathrm{~ns}$ & 4.20 & 5.42 \\
\hline $\mathrm{A}$ & 0.20 & 0.40 \\
\hline $\mathrm{B} 1$ & 0.02 & 0.009 \\
\hline $\mathrm{B} 2$ & 0.005 & 0.018 \\
\hline$<\mathrm{t}>, \mathrm{ns}$ & 1.36 & 3.74 \\
\hline
\end{tabular}

In the water solution of the Açaí extract, triplet states were detected at a wavelength of $720 \mathrm{~nm}$ when excited by a pulsed Xe lamp. The duration of the long-lived state was measured with the excitation wavelength of $530 \mathrm{~nm}$ and was approximately $\mathrm{t} \sim 13 \mu \mathrm{s}$.

Thus, the generation of singlet oxygen in the solution of this extract is due to the energy transfer from the triplet-excited states of the extract molecules upon pulsed photoexcitation with a wavelength of $530 \mathrm{~nm}$.

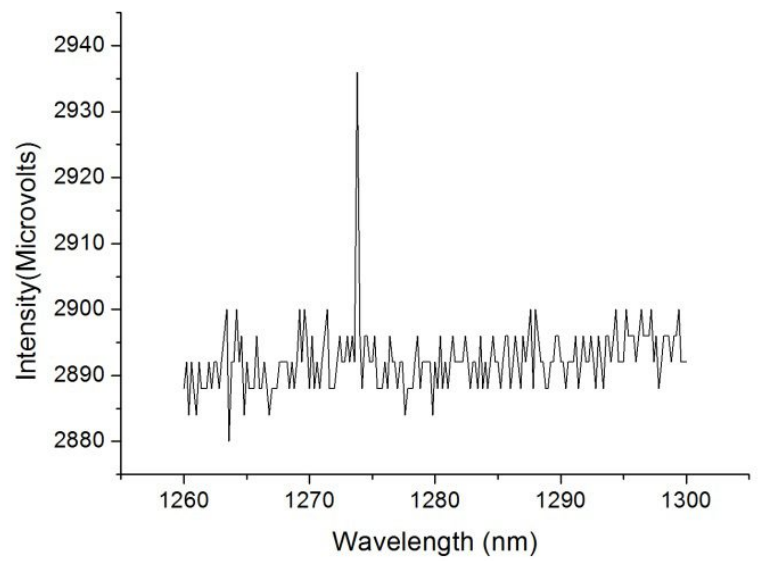

Fig.5 Luminescence spectrum of singlet oxygen under excitation at wavelength $530 \mathrm{~nm}$

In the conditions of the existence of long-living states in the molecules of the Açaí extract under excitation at wavelength $530 \mathrm{~nm}$, it is possible to assume that the generation of singlet oxygen in the solvent cell can proceed according to the classical mechanism of generation of the active form of oxygen in the ${ }^{1} \Delta_{\mathrm{g}}$ state.

In accordance with known processes of interaction in collision molecular complexes consist of oxygen and excited molecules of the sensitizer can be written:

$$
\begin{gathered}
T+{ }^{3} \Sigma_{g}^{-} \rightarrow\left(T \ldots{ }^{3} \Sigma_{g}^{-}\right)^{1,3,5} \rightarrow S_{0}+{ }^{1} \Delta_{g} \\
T+{ }^{1} \Delta_{g} \rightarrow\left(T \ldots{ }^{1} \Delta_{g}\right)^{3} \rightarrow S_{1}+{ }^{3} \Sigma_{g}^{-} .
\end{gathered}
$$

Processes (3) and (4) are realized because of diffusion contact between a molecule of a sensitizer and an oxygen molecule in a solvent. As a result of electron energy transfer from the triplet states of the sensitizer molecules of singlet oxygen in ${ }^{1} \Sigma_{\mathrm{g}}{ }^{+}$are formed with subsequent relaxation in ${ }^{1} \Delta_{\mathrm{g}}{ }^{-}$a state that emits photons of luminescence at wavelength $\lambda$ $\approx 1272 \mathrm{~nm}$ (Figure 5). The molecules of singlet oxygen formed in the ${ }^{1} \Delta_{\mathrm{g}}$ state, in the process (3), which did not emit quanta of luminescence, diffusing to triplet-excited molecules of the sensitizer (Asai) in the solvent cell form a collisional contact complex $\left(\mathrm{T} \ldots{ }^{1} \Delta_{\mathrm{g}}\right)^{3}$ and generate singlet-excited sensitizer molecules (singlet-triplet annihilation STA) in reaction (4). The collision complex with multiplicity $\left(T_{\ldots} \ldots{ }^{1} \Delta_{\mathrm{g}}\right)^{3}$ in accordance with the Wigner rule splits into excited triplet states of the sensitizer and the oxygen molecule in ${ }^{3} \Sigma_{\mathrm{g}}{ }^{-}$ground state. 


\section{CONCLUSIONS}

Thus, a candidate to photosensitizers to the PDT is proposed based on the aqueous extract from the fruit of the Amazon palm Euterpe olerácea (Açaí). For the first time directly shown that singlet oxygen is generated, among the reactive oxygen species (ROS) formed when the plant extract is irradiated with red light, which is, probably, the main responsible element for the phototoxicity of this extract.

It is also shown that singlet oxygen can be excited red and green lights by two different activation mechanisms.

\section{REFERENCES}

[1] Kudinova, N.V., Berezov, T.T., "Photodynamic therapy: search for ideal photosensitizer, " Biomed Khim. 55(5), 558-569 (2009).

[2] Sosnowska, J. Balslev, H., "American palm ethnomedicine: A meta-analysis, "J. Ethnobiol. Ethnomed., 5:43, 1-11 (2009).

[3]Wilson, B.C., Patterson M.S., "The physics, biophysics, and technology of photodynamic therapy, " Physics in Medicine and Biology 53 (9), 61-109 (2008).

[4] Monge-Fuentes V., Muehlmann, L.A., Longo, J.-P. F., Rodrigues Silva, J., Fascineli, M.L., de Souza, P., Faria, F., Degterev, I.A., Rodriguez, A., Pirani Carneiro, F.,. Lucci, C.M , Escobar, P., Amorim, R.F.B., Azevedo. R.B., "Photodynamic therapy mediated by acai oil (Euterpeoleracea Martius) in nanoemulsion: A potential treatment for melanoma, " J. Photochem.Photobiol. B: Biology 166, 301-310 (2017).

[5] Mosmann T. et al., "Rapid colorimetric assay for cellular growth and survival: application to proliferation and cytotoxicity assays, " Journal Of Immunological Methods 65 (1-2), 55-63 (1983).

[6]Cory, A.H., Owen, T.C., Barltrop, J.A., Cory, J.G., "Use of an aqueous soluble tetrazolium/formazan assay for cell growth assays in culture, " Cancer Communications 3(7), 207-212 (1991) 